\title{
有机催化在不对称构建含氮杂环化合物中的应用
}

\author{
郑雨谢珍珍陈凯向㙋月* 阳华* \\ (中南大学化学化工学院 长沙 410083)
}

\begin{abstract}
摘要 含氮杂环结构广泛存在于天然产物及药物分子中, 具有广谱的生物和药理活性, 受到有机化学家及药物化学家 们的高度关注. 开发其绿色、高效的合成方法, 尤其是不对称合成方法一直是热门研究课题. 近年来, 基于脯氨酸等氨 基酸手性模块设计合成的系列有机小分子催化剂不断被报道, 为不对称构建含氮杂环化合物提供了高效快捷的途径. 综述了我们研究小组在有机催化不对称构建含氮杂环化合物领域的研究成果, 讨论了关键反应涉及的催化机理. 关键词＼cjkstart有机催化; 不对称合成; 含氮杂环; 脯氨酸; 焦谷氨酸
\end{abstract}

\section{Application of Organocatalysis in Asymmetric Construction of Nitrogen-Containing Heterocyclic Compounds}

\author{
Zheng, Yu Xie, Zhenzhen Chen, Kai Xiang, Haoyue* Yang, Hua* \\ ( College of Chemistry and Chemical Engineering, Central South University, Changsha 410083)
}

\begin{abstract}
Nitrogen-containing heterocyclic structures are widely encountered in natural products and pharmaceuticals, which demonstrate broad-spectrum biological and pharmacological activities, and have attracted intensive attention from organic chemists and medicinal chemists. As a consequence, developing their green and efficient pathways, especially in asymmetric fashion, has always been a hot research field in the synthetic chemistry. In recent years, a series of organocatalysts based on amino acid such as proline and pyroglutamic acid have been reported, which have been well applied in the asymmetric construction of various nitrogen-containing compounds. This account mainly focuses on the research of asymmetric synthesis of nitrogen-containing heterocyclic compounds in our group, and the possible mechanisms of some typical reactions are thus discussed.
\end{abstract}

Keywords organocatalysis; asymmetric synthesis; N-heterocycle; proline; pyroglutamic acid

含氮杂环化合物广泛存在于天然产物和药物分子 中 (Scheme 1) $)^{[1]}$, 如 Cipargamin ${ }^{[1 \mathrm{cc}]}$, Martinellic ${ }^{[1 \mathrm{~d}]}$ 和 (一)-Alloaristoteline ${ }^{[1 \mathrm{e}]}$, 具有多种生物和药理活性. 据统 计, $2015 \sim 2018$ 年期间, 美国食品药品监督管理局 (FDA)批准的小分子药物结构中 $85 \%$ 以上含有含氮杂环 骨架 ${ }^{[2]}$, 而且, 许多含氮杂环药物分子包含一个或多个 手性中心, 具有不同旋光活性的异构体在生物活性上通 常显示很大的差异性. 例如, 广为人知的抗妊娠呕吐反 应药物沙利度胺, 因其对映异构体的致畸性作用导致现 代医学史上最大的灾难一一反应停事件 ${ }^{[3]}$. 再如, $(R)$ (一)-巴比妥是一种安眠药, 而其对映体会导致癫㾁 ${ }^{[4]}$. 精准、快捷且绿色、高效地构建手性含氮杂环化合物对 药物开发具有非常重要的意义.
2000 年以来, 在 $\mathrm{MacMillan}^{[5]}$ 、 $\mathrm{List}^{[6]}$ 、邓力 ${ }^{[7]}$ 、史 一安 ${ }^{[8]} 、$ Jacobsen $^{[9]}$ 等国内外有机化学家们的推动下, 有 机小分子不对称催化(本文简称有机催化)的研究取得前 所未有的发展, 为含氮杂环化合物的不对称构建提供了 全新的合成策略. 相较于传统金属催化而言, 有机催化 的反应无需担心产品中的金属残留，通常对水和空气耐 受性好，具有环境友好、反应条件温和、对底物的耐受 性好、立体选择性高以及操作简单等诸多优点. 鉴于有 机催化这些独特的优点和新型的反应性，其在不对称构 建含氮杂环骨架中的优势日益彰显.

我们课题组一直致力于开发廉价、绿色、高效的有 机催化体系以实现含氮杂环骨架的不对称构建. 本研究 专题主要总结了研究小组近年来在有机催化剂的设计、

\footnotetext{
*Corresponding authors. E-mail: hyangchem@csu.edu.cn; xianghaoyue@csu.edu.cn Received August 20, 2020; revised September 22, 2020; published online October 10, 2020. Project supported by the National Natural Science Foundation of China (Nos. 21576296, 21776318, 81703365). 国家自然科学基金(Nos. 21576296, 21776318, 81703365)资助项目.
} 


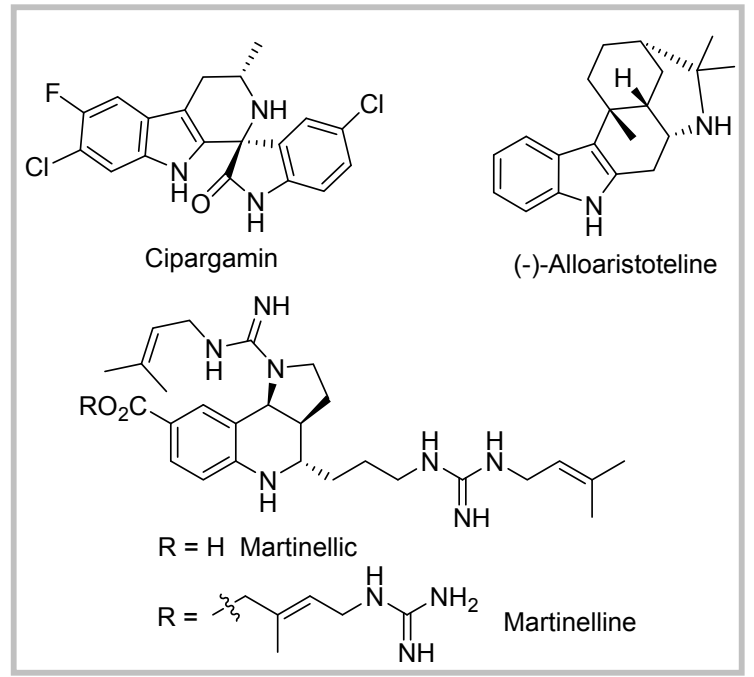

图式 1 几种典型的手性含氮杂环化合物

Scheme 1 Representative chiral nitrogen-containing heterocyclic compounds

合成及应用中取得的研究进展, 开发了一系列绿色、高 效的有机催化体系, 实现了喹啉、(螺)氧化吲哚、四氢 咔唑等重要含氮杂环化合物的不对称合成.

\section{1 基于脯氨酸有机催化剂的设计与发展}

有机小分子手性催化剂作为有机催化的关键和核 心，直接决定了反应的效率和选择性. 虽然已有大量有 机小分子催化剂先后被报道，包括氨基酸、手性膦酸、 氮杂卡宾类、手性醇类以及 2005 年 Macmillan 报道的 二级胺类催化剂 2 -羧酸二氢吲哚等 ${ }^{[10]}$, 但简易、廉价易 得的 “理想手性催化剂”仍然十分有限. 基于来源广泛 的天然手性源，如氨基酸、生物碱、糖类等与异硫氰酸 酯、方酸脂、羒酸等受体结合, 是有机催化的主要研究 方向. 其中, 脯氨酸作为一种天然二级氨基酸, 其碱性 吡咯结构单元能够与醛酮羰基结合, 而羧酸官能团可与 底物形成良好的氢键, 进一步衍生化. 脯氨酸的这些特 性使其在有机小分子不对称催化领域得到广泛应用 ${ }^{[11]}$. 为了匹配不同底物结构的特异性, 有机化学家针对脯氨 酸进行了结构修饰以改善其催化性能, 如 Jorgensen 等 ${ }^{[12]}$ 发展了系列经典的脯氨酸衍生的脯氨醇硅醚类催化
剂，已成为目前催化醛类底物参与的反应常用的有机小 分子催化剂; Melchiorre 等 ${ }^{[13]}$ 基于脯氨酸发展了二氟取 代的二级胺类催化剂, 这类催化剂能够催化经典的脯氨 醇硅醚类催化剂不能实现的反应，是对经典的二级胺类 催化剂的补充; Arvidsson 等 ${ }^{[14]}$ 首次将脯氨酸中的羒酸官 能团替换为四唑基团，成功地将其应用于不对称 Aldol 反应中，并表现出明显优于脯氨酸的催化活性. 尽管如 此，已报道的脯氨酸衍生物溶解性差，在催化反应时大 多需要使用极性溶剂 ${ }^{[15]}$, 如二甲基亚砜(DMSO)和 $N, N$ 二甲基甲酰胺(DMF), 以提高催化剂的溶解度, 但同时 增加了产物分离纯化的难度. 此外，这些催化剂的合成 需要昂贵的原料或试剂, 而且步骤繁琐. 针对以上问题, 阳华、Carter 及其同事 ${ }^{[16]}$ 首次将带有长链侧臂的十二烷 磺酰基与脯氨酸骈合，设计了新型的脯氨酸磺酰胺催化 剂 Hua Cat (Scheme 2). 该催化剂以市售的对十二烷基 磺酰氯和 $D$-或 $L$-型 $\mathrm{Cbz}$ 保护的脯氨酸为原料进行制备. 研究发现, 与传统的脯氨酸衍生物催化剂相比, Hua Cat 在非极性溶剂中的溶解性更佳，可更广泛地应用于非极 性溶剂体系中，在有机催化领域显示出了较好的应用前 景.

为了验证催化剂的催化活性, Hua Cat 先后被应用 于不对称 $\mathrm{Aldol}^{[16]}$ 反应和 $\mathrm{Mannich}^{[17]}$ 反应中. 在 1.0 equiv.水的存在下, 以 1,2-二氯乙烷为溶剂或在无溶剂 状态下, Hua Cat 可有效催化酮 $\mathbf{1}$ 和醛 $\mathbf{2}$ 之间的 Aldol 反 应(Scheme 3) ${ }^{[16]}$, 该反应条件温和且立体选择性高. 随 后，该催化剂被进一步证明能够有效催化 Mannich 反应, 以高对映选择性(up to $99 \%$ ee) 和非对映选择性(up to> $20: 1 d r)$ 得到目标产物 ${ }^{[17]}$. 该催化剂的成功开发为后续 基于脯氨酸衍生物有机催化体系的探索提供了新思路.

在众多天然氨基酸中，焦谷氨酸与脯氨酸结构十分 相似，其对映体均廉价易得. 作为重要的手性源，焦谷 氨酸在有机合成领域得到了应用 ${ }^{[18]}$. 叶松 ${ }^{[19]}$ 和 Smith $^{[20]}$ 课题组以焦谷氨酸为原料，设计合成了一系列 $N$-杂环 卡宾催化剂。该催化剂在活化烯醛和醛方面表现了优良 的催化性能. 然而, 直接利用焦谷氨酸母体结构设计有 机催化剂的报道则十分罕见．通过对焦谷氨酸的结

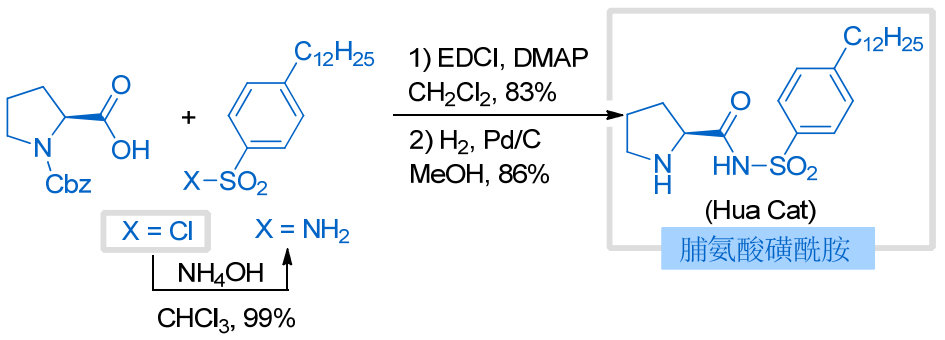

图式 2 脯氨酸磺酰胺催化剂-Hua Cat 的合成

Scheme 2 Synthesis of prolinosulfonamide catalyst-Hua Cat 


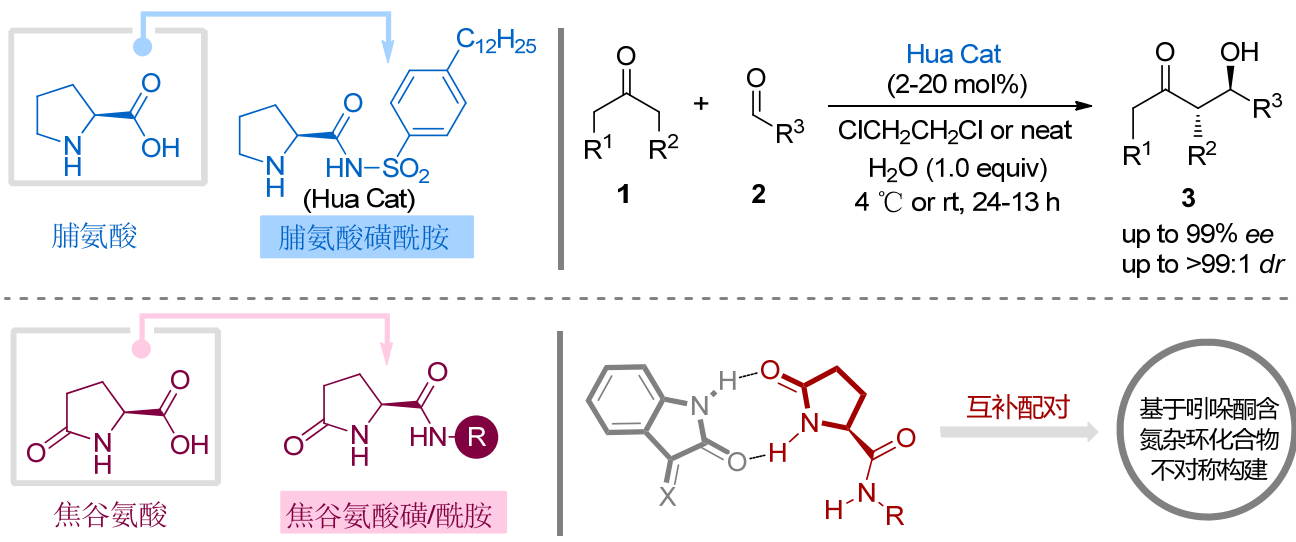

图式 3 氨基酸衍生物手性催化剂设计

Scheme 3 Design of chiral catalysts from amino acid derivatives

构分析, 可以推测焦谷氨酸的五元内酰胺结构可以同时 作为氢键的供体和受体, 有望通过形成双氢键针定底 物, 从而实现立体控制; 此外, 羧酸单元易于衍生化, 可灵活调节催化剂的活性, 便于催化剂的设计与结构优 化. 受到 Hua Cat 催化剂的启发, 我们进一步设计了新 型焦谷氨酸磺酰胺催化剂, 该催化剂可通过简单的一步 缩合反应直接制备, 在活化吲哚酮类底物方面显示出了 优异的催化性能.

\section{2 不对称构建含氮杂环}

\section{1 脯氨酸衍生物催化环己烯酮参与的含氮杂环不对 称构建}

环己烯酮是一类重要的有机合成中间体，因其结构 中含有酮羰基和环内双键而具有独特的反应活性, 常常 被用于构建各类环体系 ${ }^{[21]}$. 受到前期工作的启发 ${ }^{[16-17]}$, 我们推测 Hua Cat 系列催化剂可与环己烯酮 4 作用, 原 位脱水形成烯胺或亚胺以活化底物(Scheme 4). 基于这 一策略, 我们研究小组以环已烯酮为原料, Hua Cat 为催 化剂开发了一系列高对映选择性构筑含氮杂环化合物 的方法.

2.1.1 环已烯酮和芳基亚胺合成不对称氮杂双环 [2,2,2]辛烷骨架

氮杂双环 $[2,2,2]$ 辛烷存在于许多天然生物碱中, 因 其具有广谱的生物活性而受到合成化学家们的关注 ${ }^{[22]}$. 目前, 氮杂双环的对映选择性合成主要依赖于价格相对 昂贵的手性磷酸催化, 但所得产物的 endo/exo 选择性通 常只有 $3 / 1 \sim 4 / 1$, 且对映选择性较低 $(76 \% \sim 88 \% e e)^{[23]}$. 因此, 迫切需要开发高对映选择性、endo/exo 选择性的 合成策略来构建这类氮杂双环.

2009 年, 在无溶剂状态下, 以 Hua Cat 为催化剂, 我们研究小组实现了环已烯酮 $\mathbf{4}$ 和芳香亚胺 5 的不对称 $[4+2]$ 反应，高效构建了一系列氮杂双环 $[2,2,2]$ 辛烷 6
(Eq. 1) ${ }^{[24]}$. 该反应的对映选择性以及 endo/exo 选择性均 十分优异 $(>99 ： 1$ endolexo, up to $99 \% e e$ ). 不同取代的 芳基亚胺在该反应条件下均具有良好的耐受性，含吸电 子基团的底物通常以适中的产率和高对映选择性得到 目标产物; 然而给电子基团会使亚胺的亲电性降低, 导 致反应产率和对映选择性略有降低. 在该反应中, Hua Cat 中的磺酰胺氮氢与亚胺之间形成氢键活化亚胺，与 此同时，其吡咯结构与环己烯酮作用脱水异构成烯胺， 并从亚胺的 $S i$ 面进行亲核进攻获得相应的氮杂双环 $[2,2,2]$ 辛烷产物.

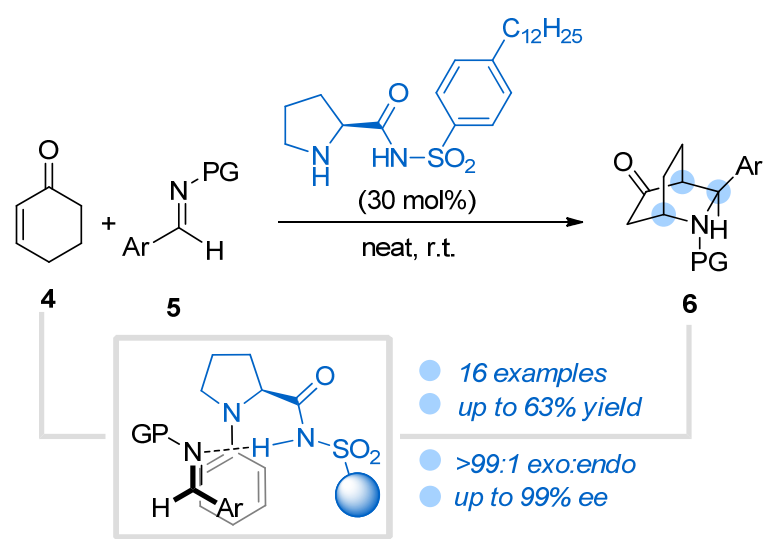

2.1.2 环已烯酮参与不对称 1,3-偶极环加成反应手 性构建氧化吲哚骨架

含有螺环氧化吲哚骨架的化合物很多都具有较高 的生物活性，包括抗炎、抗糖尿病、抗肿瘤和乙酰胆碱 酷酶抑制活性等 ${ }^{[25]}$. 在过去的几十年中, 开发结构多样 和立体控制的方法以合成螺环氧化吲哚一直是许多科 学家追求的目标 ${ }^{[26]}$. 然而, 已报道的合成方法中以非手 性合成为主，或者依赖于使用手性分子作为原料以构建 手性螺环氧化吲哚. 因此, 螺环氧化吲哚的不对称合成 仍然面临着巨大的挑战. 近年来, 通过亚胺叶立德与缺 电子的烯烃进行 1,3-偶极环加成反应来制备螺氧化吲哚 


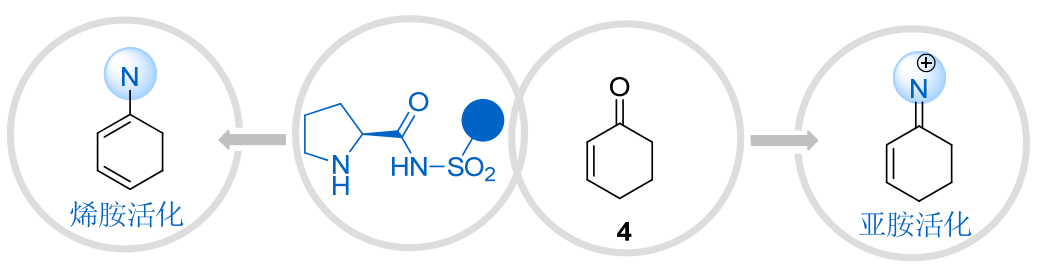

图式 4 Hua Cat 催化剂对环已烯酮的活化模式

Scheme 4 Activation mode of cyclohexenone by Hua Cat

得到了快速发展 ${ }^{[27]} .2012$ 年, 龚流柱课题 ${ }^{[28]}$ 组通过有机 不对称催化方法成功合成了一系列含有多个连续季碳 中心的螺氧化吲哚, 这是第一个基于亚胺叶立德通过不 对称 1,3-偶极环加成合成手性螺环吲哚的例子. 此后, 许鹏飞 ${ }^{[29]}$ 和屠树江 ${ }^{[27]}$ 课题组通过有机催化 1,3 -偶极环 加成策略成功制备了螺环吲哚. 值得注意的是, 这些策 略中使用的烯烃多为线性烯烃(Eq. 2). 环状烯烃特别是 反应性较低的环状 $\alpha$-烯酮与亚胺叶立德的 1,3-偶极环加 成反应却罕见报道. 因此迫切需要开发一种基于环状烯 酮的 1,3-偶极环加成反应, 以发展新型高效的不对称合 成方法来合成手性螺环氧化吲哚骨架.

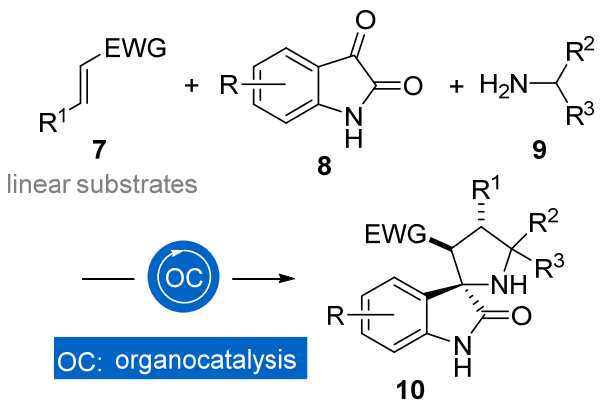

2014 年, 我们采用 Hua Cat 催化剂实现了吲哚酮 8/ 氨基丙二酸酯 9 原位生成的亚胺叶立德与环己烯酮 4 之 间的不对称 1,3-偶极环加成反应, 成功地构建了结构新 渘的多环手性螺吲哚环化合物 12 (Eq. 3) $)^{[30]}$. 在该反应 过程中, 我们推测 Hua Cat 上的吡咯结构与环已烯酮 4 结合脱水生成亚胺, 侧臂上磺酰胺的 $\mathrm{N}-\mathrm{H}$ 可以与被三 乙胺去质子化的吲哚酮亚胺叶立德中的氮原子与氧原 子形成氢键, 从而控制反应的立体选择性. 原位形成的 叶立德从纸面上与亚胺进行 1,3-偶极环加成反应, 以最 高 95\%的化学收率，>20：1 的非对映体选择性和 $99 \%$ 的 $e e$ 值得到目标产物.

后续, 为了拓展该方法学的实用性, 进行了一系列 合成转化实验. 将产物经过一步还原或两步脱羧, 以较 好的收率和高对映选择性转化为结构多样的新型螺环 氧化吲哚衍生物(Scheme 5a, 5b). 此外, 产物可以通过 Fisher 吲哚合成法一步构建具有吲哚和靛红骨架的多环 杂合体 14 (Scheme 5c).

在此基础上, 将 1,3-偶极子更换为富电子的共轭双
烯 2-吲哚烯 15, 将其与环状烯酮 4 通过 exo-Diels-Alder 环加成反应，一步快捷构建具有三个连续立体中心的四 氢咔唑结构 16 (Eq. 4) ${ }^{[31]}$. 值得一提的是, 在该反应体系 中, $(S)$-樟脑磺酸可以通过氢键传递作用提高非对映体 选择性和对映体选择性. 推测(S)-樟脑磺酸上的磺酸根 与 2-吲哚烯上的氮氢以及催化剂上的磺酰胺活性质子 形成氢键, 从而控制 2-吲哚烯从 $S i$ 面进攻催化剂与环己 烯酮 4 生成的亚胺. 该反应能简便、高效、高立体选择 性 (exolendo $>20 ： 1 d r, 90 \sim>99 \%$ ee ) 地得到一系列结 构新颖的多环螺氧化吲哚衍生物, 仅通过简单的重结晶 就能快速分离得到目标产物, 无需经过柱层析分离, 因 而具有很好的应用前景.

类似地，上述反应中生成的四氢咔唑骨架 16a 可进 一步通过 Fischer 吲哚合成法以高收率 (78\%)和高对映体 选择性( $>99 \% e e,>20: 1 d r)$ 构建结构复杂的全新多 环化合物 17. 此外, 产物 17 中的溴原子有助于分子内 偶联反应，进一步环化以优异的立体选择性(>20：1 $d r,>99 \% e e$ ) 构建多环四氢咔唑 $\mathbf{1 8}$ (Scheme 6), 有效实 现了不同官能团化螺环氧化吲哚衍生物的合成.

2.1.3 环已烯酮参与不对称 Michael 加成反应合成叫 内酯衍生物

$\alpha, \alpha$-二取代的 $\alpha$-氨基酸是一类非常有用的手性化合 物, 但是其对映选择性合成充满挑战 ${ }^{[32]}$. 叮内酯是一类 含氮杂环结构的内酯, 可作为 Michael 供体参与多种化 学转化, 同时也可作为合成手性 $\alpha$-氨基酸的前体 ${ }^{[33]}$. 王 锐 ${ }^{[34]}$ 、叶金星 ${ }^{[35]}$ 和王兴旺 ${ }^{[36]}$ 等课题组在吅内酯参与的对 映选择性反应研究方面做出了卓越贡献. 对于亲电性较 低的反应底物，如易于烯醇化的芐叉丙酮和环己烯酮， 有机催化对映选择性共轭加成反应仍然难以实现.

在 Hua Cat 的催化作用下, 我们首次实现了环己烯 酮等反应活性相对较低的 Michael 受体 19 与吅内酯 $\mathbf{2 0}$ 的对映选择性共轭加成反应, 以高对映选择性和中等至 良好的收率、高效构建了含有两个连续季碳和叔碳中心 的加合物 21 或 22 (Scheme 7) ${ }^{[37]}$, 为 $\alpha, \alpha$-二取代的 $\alpha$-氨 基酸的合成提供了新的合成策略. 在反应过程中, Hua Cat 的吡咯结构单元和烯酮缩合脱水生成亚胺离子, 而 催化剂的磺酰胺能与烯醇化的芐基吅内酯生成氢键，从 而控制吅内酯从亚胺离子的 $S i$ 面进行亲核进攻. 值得注 


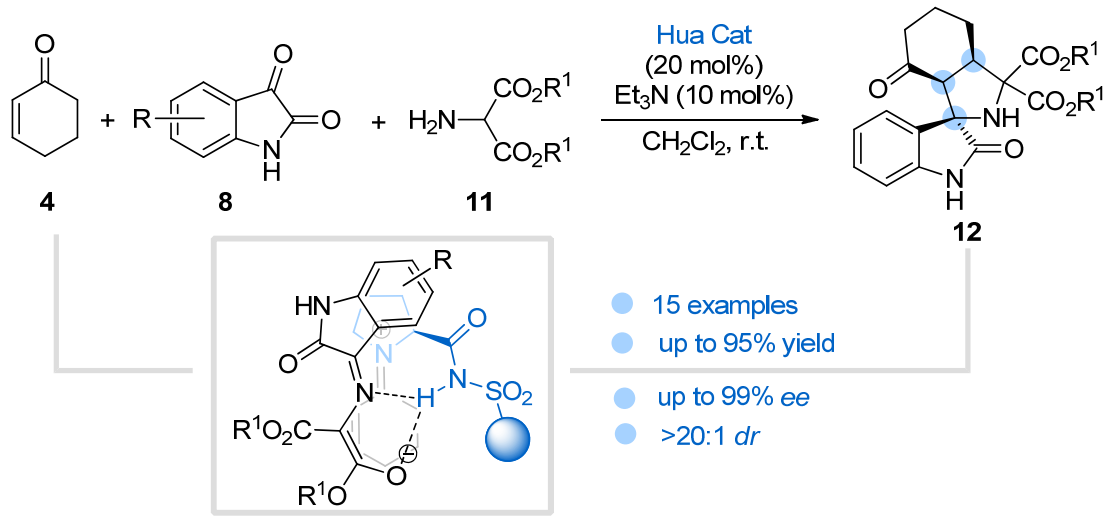

(a)
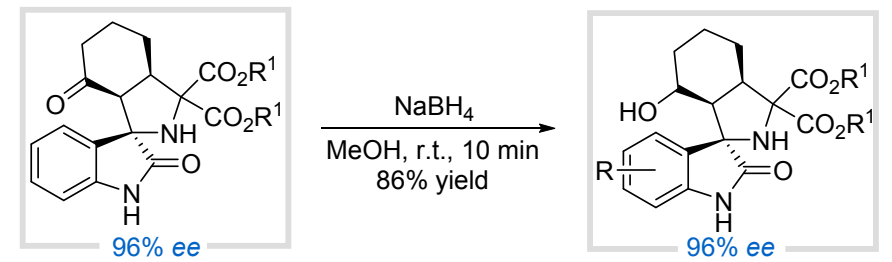

(b)

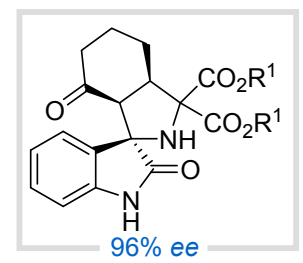

$$
\begin{gathered}
\text { (1) } \mathrm{MeOH} \text { (aq.) } \\
\text { EtOH, r.t., } 30 \mathrm{~min} \\
\hline \text { (2) toluene, } 110{ }^{\circ} \mathrm{C}, 10 \mathrm{~min} \\
87 \% \text { yield } \\
\text { exolendo } 70: 30
\end{gathered}
$$

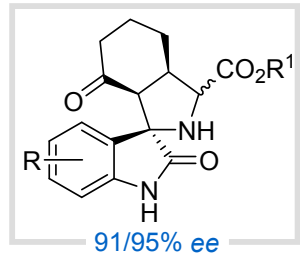

(c)
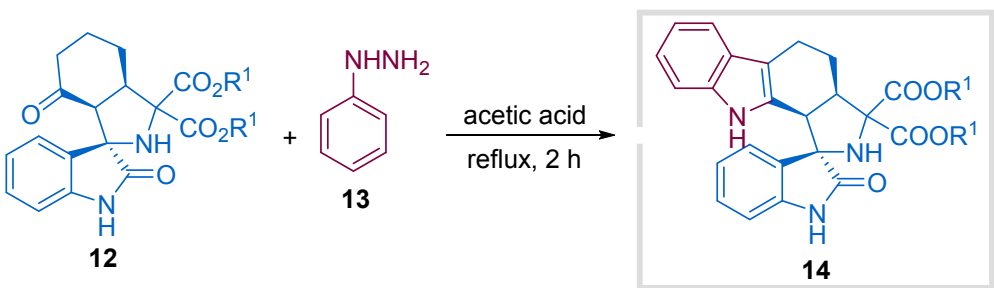

图式 5 手性螺环氧化吲哚的衍生化

Scheme 5 Transformation of chiral spirooxindole compounds

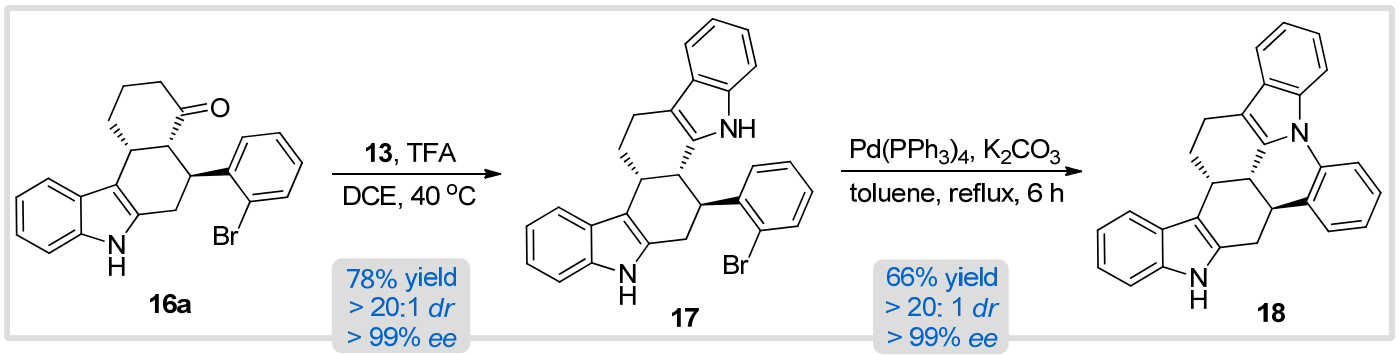

图式 6 四氢咔唑的衍生化

Scheme 6 Transformation of tetrahydrocarbazole

意的是, 反应体系中的水能通过氢键传递提升该反应的 立体控制效果, 在含氟溶剂(三氟甲苯)中反应效果最好. 所得产物通过浓盐酸水解再经过简单的重结晶可获得 $\alpha, \alpha$-二取代的 $\alpha$-氨基酸 23 (Eq. 5). 这一策略有效弥补了 已有方法底物的局限性, 拓展了唤酯在有机催化反应
中的应用.

2.1 .4 环已烯酮参与不对称 $[4+2]$ 环加成反应合成八 氢呋啶结构

具有三环骨架的鲐啶结构存在于许多具有生物活 性的天然产物中 ${ }^{[38]}$. 然而, 八氢呋啶作为听啶的一个亚 


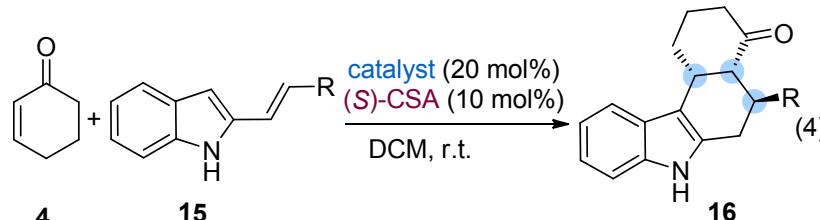

4

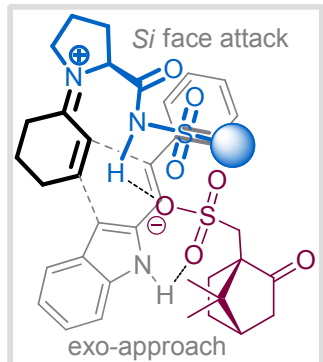

exo cycloadduct

up to $87 \%$ yield

$>99: 1 d r$

up to $>99 \%$ ee
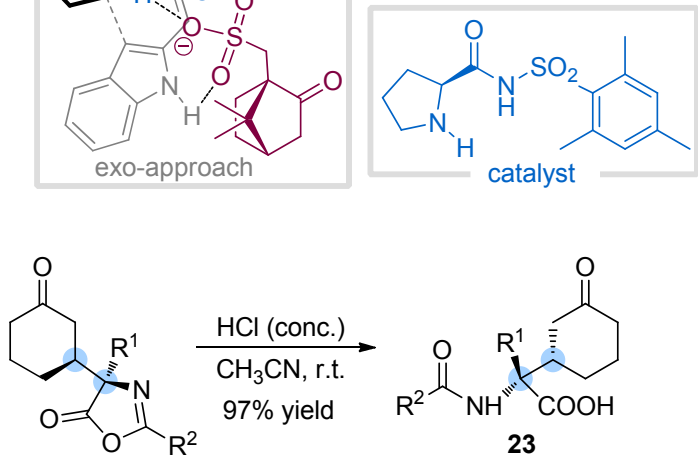

23

$22 \mathrm{a}$

$\mathrm{R}^{1}=\mathrm{Bn}, \mathrm{R}^{2}=\mathrm{Ph} ; 99.5: 0.5 \mathrm{er} ;>20: 1 \mathrm{dr}$

类, 其合成以及药理学研究都十分有限 ${ }^{[39]}$. 究其原因是 缺乏高效的构建方法, 难以快速获得结构多样的八氢听 啶化合物来进行相关的生物活性测试. 迄今为止，已有 一些合成方法可以构建八氢听啶结构，如酸催化异苯甲 酮一苯胺缩合、肟磺酸的贝克曼重排以及路易斯酸催化 的 imine-Diels-Alder 反应等, 但是关于手性八氢鲐啶的 不对称合成方法仍然十分罕见 ${ }^{[40]}$.
基于前期关于有机催化环已烯酮 4 参与不对称反应 的研究基础，我们推测八氢鲐啶骨架有望通过 $a z a$-Michael/Aldol 反应来构建. 通过对催化剂、反应溶 剂的系统篎选，最终在 4-氧二甲基叔丁基硅基脯氨酸的 催化下, 以环已烯酮 4 和邻氨基苯甲醛衍生物 24 为原 料，成功实现了含三个连续手性中心的八氢鲐啶骨架 25 的构建(Eq. 6) ${ }^{[41]}$. 该反应可以最高 $99 \%$ 的收率和 $99 \%$ 的 $e e$ 值得到目标产物，底物适用范围广. 与 Hua Cat 类 似, 催化剂中的吡咯结构单元与环己烯酮反应脱水缩合 生成活性亚胺中间体. 另一方面，邻氨基苯甲醛则在强 极性溶剂和催化剂中的酸性质子双重作用下烯醇化生 成氮杂双烯的活性中间体，并从活性亚胺的 $S i$ 面进攻发 生 $[4+2]$ 反应，从而高对映选择性地得到产物. 此外, 两个具有挑战性的反应底物环戊烯酮和环庚烯酮也适 用于该合成策略，尽管反应时间有所延长且产率也有所 降低，但进一步扩展了含氮杂环产物分子骨架的结构类

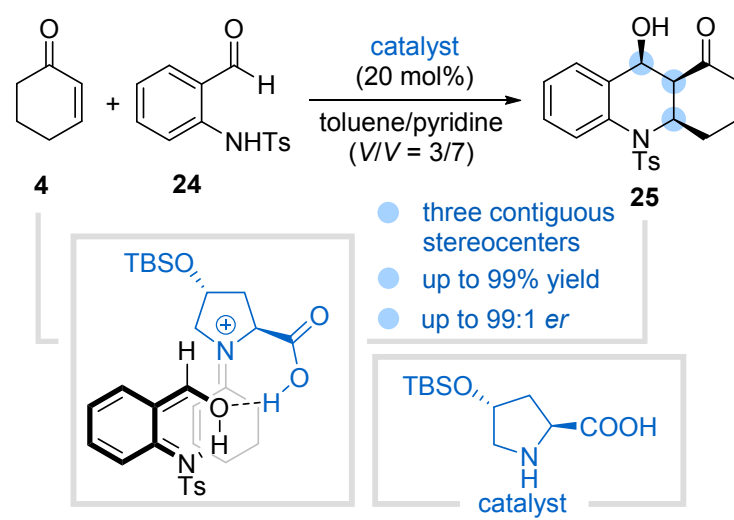

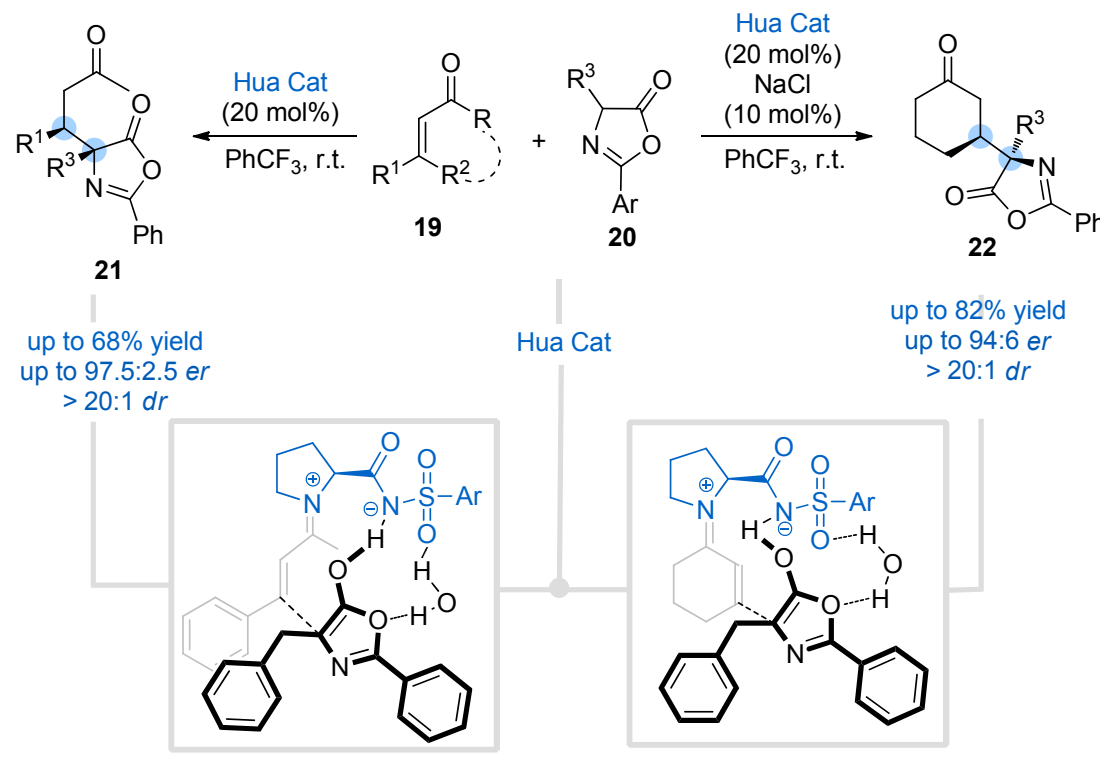

图式 7 Hua Cat 催化呋内酯不对称 Michael 加成反应

Scheme 7 Hua Cat-catalyzed asymmetric Michael addition reaction of azlactones 
型.

\section{2 焦谷氨酸衍生物催化不对称构建含氮吲哚杂环}

基于对靛红底物结构特征的分析, 我们设想通过焦 谷氨酸磺酰胺催化剂与其内酰胺结构采取 “互补配对” 模式，继而通过双氢键作用实现不对称环化反应. 2017 年, 我们利用新设计的 $L$-焦谷氨酸磺酰胺催化剂实现了 亚甲基氧化吲哚 26 和 2-吲哚烯 $\mathbf{2 7}$ 的不对称 Diels-Alder 环化反应(Eq. 7) ${ }^{[42]}$, 以高达 $99 \%$ 的对映选择性和 $>20$ ： 1 的非对映选择性合成了氧化吲哚螺四氢咔唑化合物 28, 产物中含有三个连续的手性中心, 其中含有一个或 两个季碳手性中心. 由于该体系背景反应明显, 因此低 反应物浓度是保障高对映选择性的关键.

为了深入了解该催化剂在此反应中所扮演的角色, 我们进行了一系列控制实验 (Scheme 8). 首先, $N$-保护 的吲哚烯和/或 $N$-保护的亚甲基氧化吲哚在标准条件下 进行反应, 反应的对映体选择性显著降低. 这说明了反 应物中的 $\mathrm{N}-\mathrm{H}$ 与催化剂之间存在着氢键作用力. 进一 步的 ${ }^{1} \mathrm{H} N \mathrm{NR}$ 实验也佐证了这一猜测, 将反应物分别与 催化剂 $1: 1$ 混合后的核磁表征发现, $\mathrm{N}-\mathrm{H}$ 位移发生了 明显的改变. 最后, 研究了水对该反应的影响. 反应体 系彻底除水会导致该反应的消旋化, 而添加 10 equiv.水 则对该反应的对映体选择性及产率毫无影响. 基于上述 机理控制实验, 提出了可能的催化剂与底物之间的催化 模型(Eq. 7). 催化剂中的焦谷氨酸内酰胺结构与亚甲基 氧化吲哚的内酰胺形成双氢键, 而在 2-吲哚烯上的 $\mathrm{N}-$ $\mathrm{H}$ 通过水的氢键传递作用与催化剂中的磺酰胺模块形 成氢键.

鉴于亚甲基氧化吲哚和 3-羟基氧化吲哚具有良好 的极性匹配, 在上述工作基础上将 2-吲哚烯底物替换为 3-羟基氧化吲哚 29, 设计了环重组多米诺环化反应来构 建结构复杂的吲哚螺环骨架 31 (Eq. 8) 8 $^{[43]}$. 遗憾的是, 焦 谷氨酸系列有机催化剂在该反应体系中效果不佳, 而奎
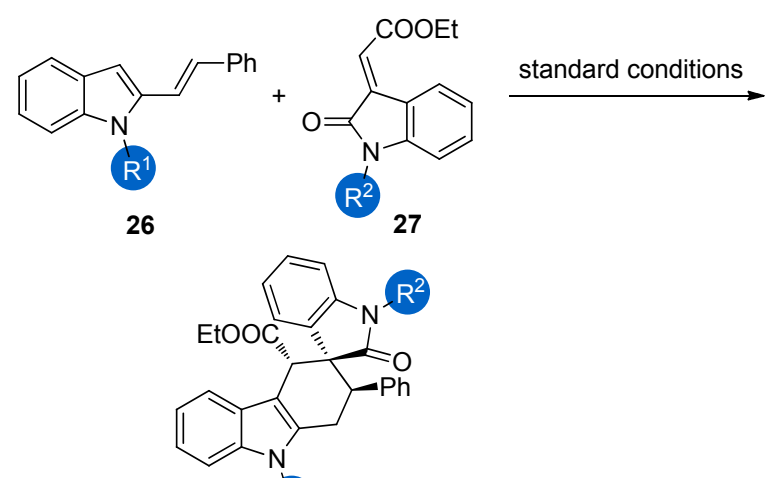

R 28
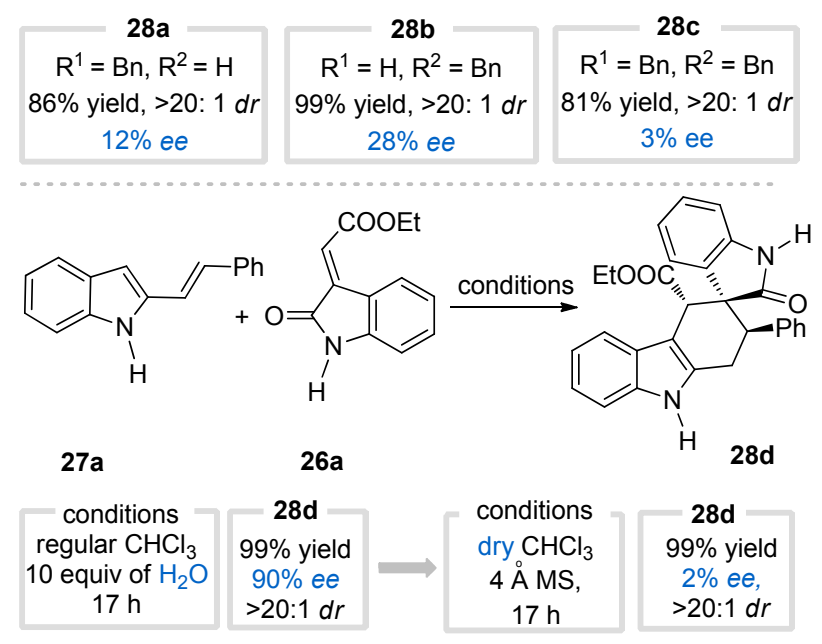

图式 8 机理研究

Scheme 8 Mechanistic studies

宁方酰胺类催化剂表现出了优异的催化性能. 在奎宁方 酰胺类催化剂的作用下, 实现了极性匹配的 3-差基氧化 吲哚和亚甲基氧化吲哚 30 的立体选择性 Michael 加成/ 分子内开环/内酰胺化 “一锅法” 串联反应. 该策略能 以较好的收率 $(81 \% \sim 97 \%)$ 、理想的对映选择性( $90 \sim>$ $99 \% e e)$ 和非对映选择性( $71 ： 29 \sim>95 ： 5 d r)$ 高效、简 便地构建含有三个连续手性中心的多环氧化吲哚螺喹 啉酮骨架. 该反应可以顺利地放大至 $1 \mathrm{mmol}$ 规模, 反<smiles>[R]C([R])=C1C(=[OH+])Nc2cc[R1]cc21</smiles>

26

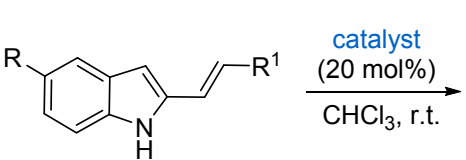

27<smiles>[R]c1ccc2c(c1)[C@]1(C(=O)N2)C([R])Cc2[nH]c3ccc([R])cc3c2[C@H]1[R]</smiles>

28 


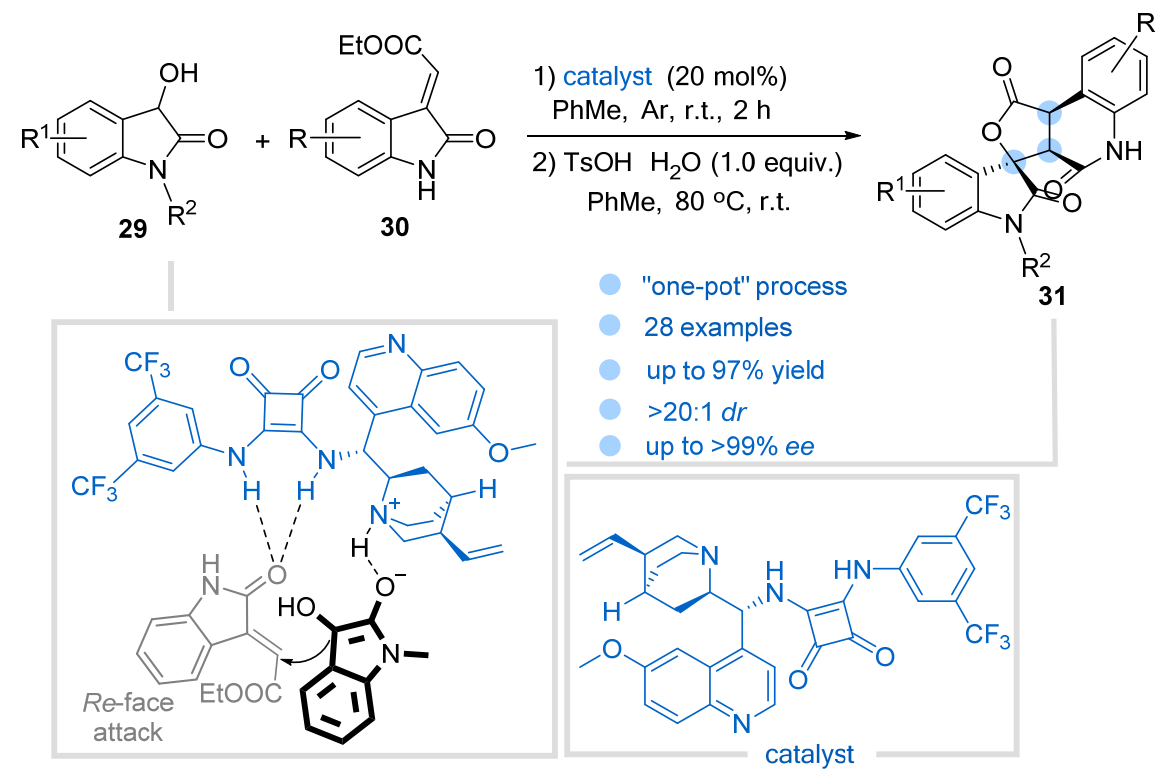

应效率几乎不受影响, 进一步证明了该策略的实用性和 可扩展性. 基于产物的绝对构型与实验结果提出了反应 过程的机理: 首先, 3-差基氧化吲哚 29 被奎宁方酰胺催 化剂的三级胺脱去质子烯醇化, 产生一个高活性的 Michael 供体; 同时, 亚甲基氧化吲哚 30 作为 Michael 受体，通过氢键的相互作用由方酰胺基团导向. 烯醇化 的 3-差基氧化吲哚 29 对亚甲基氧化吲哚 $\mathbf{3 0}$ 进行 $R e$ 面 进攻, 对映选择性地生成关键中间体, 进一步环化得到 最终产物 31.

进一步将焦谷氨酸与广泛使用的手性源 1,2-环已二 胺缩合得到另一类新型 $L$-焦谷氨酸环己二胺催化剂, 我 们发现这类催化剂可以催化环己酮 32 与吲哚酮 8 之间 的不对称 Aldol 反应(Scheme 9) ${ }^{[44]}$. 然而, 在底物耐受性 的考察中意外发现, 底物无论是连有吸电子取代基还是 供电子取代基, 在模型反应所篮选出来的最优条件下均 表现出极差的对映选择性. 为了阐明产生该现象的原 因, 我们跟踪反应过程中对映选择性的变化, 发现反应 过程初期显示出良好的对映体选择性, 但随着时间变化 产物的 ee 值逐渐降低. 基于跟踪实验结果, 并考虑到 Aldol 反应的可逆性, 可以认为立体专一性逆羟醛缩合 反应是导致 $e e$ 值随着反应时间逐渐降低的根本原因.

为了验证这一观点, 我们进一步开展了系列控制实 验. 首先, 合成了 $L$-焦谷氨酸环己二胺催化剂的对映体 ent-Cat，并用它催化该反应得到了产物的对映体 ent-33. 分别将催化剂 Cat 和 ent-Cat 与两种产物 33 和 ent-33 在 DMF 中反应 $1 \mathrm{~h}$, 实验结果显示, 催化剂 Cat 只能降低产 物 33 的 $e e$ 值而不会影响产物 ent-33, 与此相对应的是 $e n t$-Cat 只能降低产物 ent-33 的 ee 值. 这一现象充分表 明在该条件下, 手性催化剂只能单一催化一种构型产物

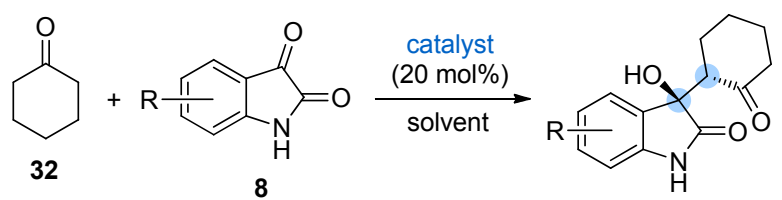

33

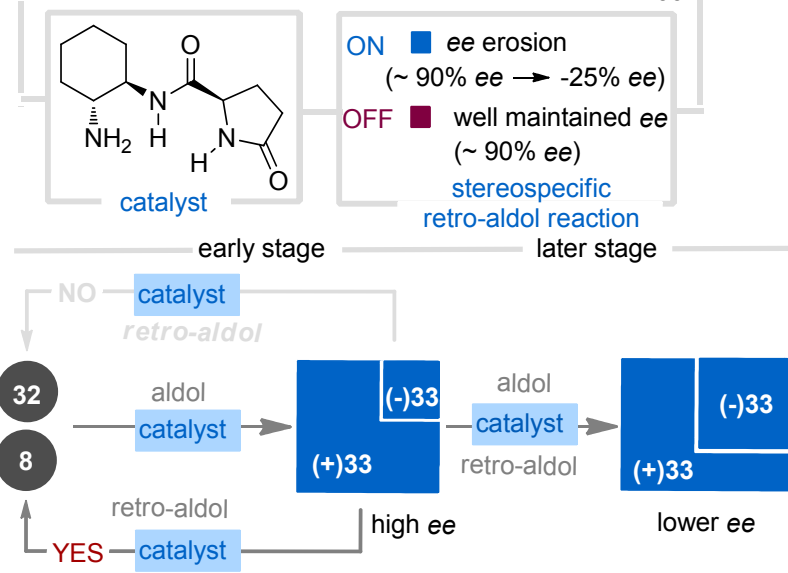

图式 9 新型 $L$-焦谷氨酸环己二胺催化剂催化合成手性 3-羟 基吲哚 33

Scheme 9 Synthesis of chiral 3-hydrooxindole 33 catalyzed by novel $L$-pyroglutamic acid derivatives

的逆反应. 因此，优势构型产物不断地发生逆 Aldol 反 应导致产物减少, 弱势构型产物则稳定地增加, 最终造 成产物 33 的对映体选择性不断降低. 采取环已酮作为 溶剂则可以有效地抑制逆反应，从而高对映体选择性地 合成目标产物 33. 毫无疑问, 这一现象的发现将加深我 们对不对称可逆反应的理解，为提升不对称合成中催化 体系的手性控制提供了一种全新的视角.

\section{3 总结与展望}

综上所述，有机催化在不对称构建含氮杂环中发挥 
了重要的作用. 综述了我们研究小组基于有机小分子催 化中二级胺催化的反应模式和机理, 发展了系列源于廉 价易得的手性源脯氨酸及焦谷氨酸的二级胺类新催化 剂, 并利用这类催化剂开发了一系列高对映选择性构筑 含氮杂环的方法, 主要总结了喹啉、(螺)氧化吲哚、四 氢咔唑等重要含氮杂环化合物的不对称合成, 这些研究 工作丰富了有机小分子催化的反应类型和含氮化合物 的合成途径. 可以预见, 随着对于新型有机催化剂的研 制以及高效合成途径的开发, 将进一步丰富手性含氮杂 环化合物库, 为药物发现提供坚实的结构基础.

\section{作者简介}

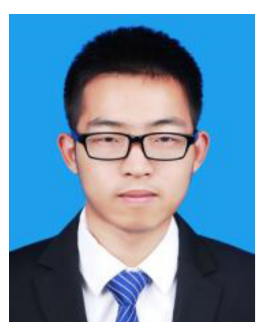

郑雨, 2012 2016 年就读于中南大学化学化工学 院, 获得学士学位; 2016 2019 年就读于中南大学化学 化工学院, 获得硕士学位; 2019 年至今在中南大学化学 化工学院阳华课题组攻读博士学位. 主要研究方向为有 机小分子催化及新型有机小分子催化剂的设计、合成与 应用。

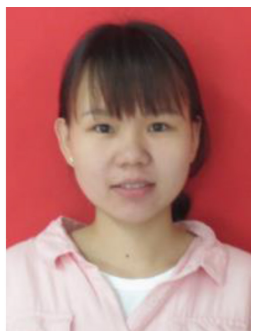

谢珍珍, 2014 2018 年就读于吉首大学化学化工学 院, 获得学士学位; 2018 年至今在中南大学化学化工学 院阳华教授课题组攻读硕士学位. 主要研究方向为有机 催化合成含氮含氧杂环.

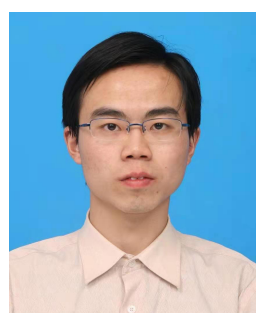

陈凯, 博士, 讲师. 2005 2009 年就读于南开大学 药学院, 获得学士学位. 2009 2014 年就读于北京大学 化学化工学院, 获得理学博士学位, 师从吴云东教授和 Olaf Wiest 教授, 在表观遗传学调控蛋白与小分子的相
互作用机制以及抑制剂设计方面取得了一定进展，对组 蛋白去乙酰化酶家族的选择性抑制提出了新的理论模 型. 2015 年加入华南理工大学化学化工学院祝诗发课题 组从事博士后工作，对过渡金属催化的烯炔醛环异构化 反应机理进行了深入的理论研究. 2019 年在韩国高等科 学技术院 Mookie Baik 课题组进行访问研究. 2019 年年 底加入中南大学化学化工学院, 主要从事基于自由基中 间体串联反应的机理研究以及新型有机催化剂的设计 开发.

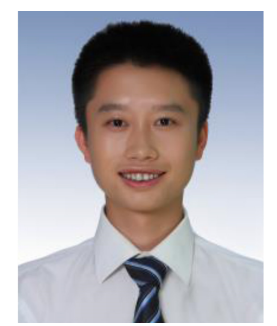

向䶋月，博士，副教授，硕士生导师. 2006 2010 年就读于湖南师范大学化学化工学院, 获得学士学位. 2010 2015 就读于中国科学院上海药物研究所, 获得 博士学位, 在杂环化合物库的构建、苗子/先导化合物的 发现取得了一定进展. 构建了基于色酮的含氮杂环小分 子化合物库, 并从中发现了一些结构新颖的抗肿瘤先导 化合物，设计合成了系 PI3K 抑制剂，在体外表现出良 好的抗肿瘤活性. 2015 年至今于中南大学化学化工学院 承担有机化学、药物化学等教学工作, 并开展研究工作. 致力于光氧化还原催化体系研究以及含氮/氟杂环小分 子化合物库构建新方法的开发.

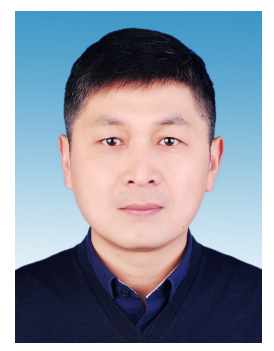

阳华，博士，中南大学海外高层次人才 “升华学者” 特聘教授, 博士生导师. 1991 1995 年就读于四川大学 精细化工专业, 获得学士学位; 1996 1998 年就读于四 川大学有机化学专业, 获得硕士学位; 2001 2006 就读 于美国西弗吉尼亚大学, 获得博士学位; 2006 2011 年 在美国俄勒冈州立大学开展博士后研究工作, 并随后被 提升为助理研究员独立开展研究工作; 2011 年至今以中 南大学 “升华学者” 特聘教授进入中南大学化学化工学 院任教并开展研究工作. 主要从事有机合成和催化方面 的研究, 重点在手性催化、光催化、复杂手性药物分子 的合成与改性等领域取得了系列研究成果. 


\section{References}

[1] (a) Wang, S.-Q, Huang, W.-Y, Zhang, X.-R, Zhang, X.-T, Pan. C.-X. Chin. J. Org. Chem. 2020, 40, 959 (in Chinese).

(王淑琴, 黄婉云, 张小蓉, 张晓婷, 潘成学, 有机化学, 2020, 40,959.)

(b) Li, Q.; Wang, Y.; Hu, M.-J; Chen, P.; You, W.-W; Zhao, P.-L. Chin. J. Org. Chem. 2017, 37, 967 (in Chinese).

(黎秋, 汪雨, 胡孟金, 陈鹏, 游文玮, 赵培亮, 有机化学, 2017, 37, 967.)

(c) White, N. J.; Pukrittayakamee, S.; Phyo, A. P.; Rueangweerayut, R.; Nosten, F.; Jittamala, P.; Jeeyapant, A.; Jain, J. P.; Lefevre, G.; Li, R.; Magnusson, B.; Diagana, T. T.; Leong, F. J. N. Engl. J. Med. 2014, 371, 403 .

(d) Witherup, K. M.; Ransom, R. W.; Graham, A. C.; Bernard, A. M.; Salvatore, M. J.; Lumma, W. C.; Anderson, P. S.; Pitzenberger, S. M.; Varga, S. L. J. Am. Chem. Soc. 1995, 117, 6682.

(e) Güller, R.; Borschberg, H.-J. Tetrahedron: Asymmetry 1992, 3, 1197.

[2] Vitaku, E.; Smith, D. T.; Njardarson, J. T. J. Med. Chem. 2014, 57, 10257.

[3] Sun, G.; Zhang, W.-W; Zhan, X.-P; Liu, Z.-L; Mao. Z.-M. Chin. J. Org. Chem. 2014, 34, 546 (in Chinese). (孙广龙, 张蔚蔚, 詹晓平, 刘增路, 毛振民, 有机化学, 2014, $34,546$.

[4] Smith, M. C.; Riskin, B. J. Drugs 1991, 42, 365.

[5] (a) Jui, N. T.; Garber, J. A.; Finelli, F. G.; MacMillan, D. W. J. Am. Chem. Soc. 2012, 134, 11400.

(b) Kwiatkowski, P.; Beeson, T. D.; Conrad, J. C.; MacMillan, D. W. J. Am. Chem. Soc. 2011, 133, 1738.

[6] (a) Schreyer, L.; Properzi, R.; List, B. Angew. Chem. Int. Ed. 2019, $58,12761$.

(b) Ouyang, J.; Kennemur, J. L.; De, C. K.; Fares, C.; List, B. J. Am. Chem. Soc. 2019, 141, 3414.

[7] (a) Hu, B.; Bezpalko, M. W.; Fei, C.; Dickie, D. A.; Foxman, B. M.; Deng, L. J. Am. Chem. Soc. 2018, 140, 13913.

(b) Tian, S.-K.; Chen, Y.; Hang, J.; Tang, L.; McDaid, P.; Deng, L. Acc. Chem. Res. 2004, 37, 621.

[8] (a) Zhu, Y.; Wang, Q.; Cornwall, R. G.; Shi, Y. Chem. Rev. 2014, 114,8199 .

(b) Wong, O. A.; Shi, Y. Chem. Rev. 2008, 108, 3958.

[9] (a) Li, Q.; Levi, S. M.; Jacobsen, E. N. J. Am. Chem. Soc. 2020, 142,11865 .

(b) Doyle, A. G.; Jacobsen, E. N. Chem. Rev. 2007, 107, 5713.

[10] (a) Dutartre, M.; Bayardon, J.; Juge, S. Chem. Soc. Rev. 2016, 45, 5771.

(b) James, T.; van Gemmeren, M.; List, B. Chem. Rev. 2015, 115, 9388 .

(c) Melchiorre, P.; Marigo, M.; Carlone, A.; Bartoli, G. Angew. Chem. Int. Ed. 2008, 47, 6138 .

(d) Enders, D.; Niemeier, O.; Henseler, A. Chem. Rev. 2007, 107, 5606 .

(e) Kunz, R. K.; MacMillan. J. Am. Chem. Soc. 2005, 127, 3240.

[11] (a) Wang, L.; Liu, J. Synthesis 2016, 49, 960.

(b) Mukherjee, S.; Yang, J. W.; Hoffmann, S.; List, B. Chem. Rev. 2007, 107, 5471.

(c) List, B.; Lerner, R. A.; Barbas, C. F. J. Am. Chem. Soc. 2000, $122,2395$.

[12] (a) Reyes-Rodriguez, G. J.; Rezayee, N. M.; Vidal-Albalat, A.; Jorgensen, K. A. Chem. Rev. 2019, 119, 4221.

(b) Jensen, K. L.; Dickmeiss, G.; Jiang, H.; Albrecht, L.; Jorgensen, K. A. Acc. Chem. Res. 2012, 45, 248.

[13] Silvi, M.; Verrier, C.; Rey, Y. P.; Buzzetti, L.; Melchiorre, P. Nat. Chem. 2017, 9, 868 .

[14] Hartikka, A.; Arvidsson, P. R. Tetrahedron: Asymmetry 2004, 15, 1831.

[15] (a) Enders, D.; Grondal, C.; Huttl, M. R. Angew. Chem. Int. Ed. $\mathbf{2 0 0 7}, 46,1570$ (b) Cordova, A.; Watanabe, S.; Tanaka, F.; Notz, W.; Barbas, C. F. J. Am. Chem. Soc. 2002, 124, 1866.

[16] Yang, H.; Carter, R. G. Org. Lett. 2008, 10, 4649.

[17] Yang, H.; Carter, R. G. J. Org. Chem. 2009, 74, 2246.

[18] (a) Zaminer, J.; Brockmann, C.; Huy, P.; Opitz, R.; Reuter, C.; Beyermann, M.; Freund, C.; Muller, M.; Oschkinat, H.; Kuhne, R.; Schmalz, H. G. Angew. Chem. Int. Ed. 2010, 49, 7111.

(b) Bateman, L.; Breeden, S. W.; O'Leary, P. Tetrahedron: Asymmetry 2008, 19, 391.

[19] Chen, X.-Y.; Gao, Z.-H.; Ye, S. Acc. Chem. Res. 2020, 53, 690.

[20] (a) Davies, A. T.; Pickett, P. M.; Slawin, A. M. Z.; Smith, A. D. ACS Catal. 2014, 4, 2696.

(b) Massey, R. S.; Collett, C. J.; Lindsay, A. G.; Smith, A. D.; O'Donoghue, A. C. J. Am. Chem. Soc. 2012, 134, 20421.

[21] (a) Yarlagadda, S.; Sankaram, G. S.; Balasubramanian, S.; Subba Reddy, B. V. Org. Lett. 2018, 20, 4195.

(b) Liu, H.; Cun, L.-F.; Mi, A.-Q.; Jiang, Y.-Z.; Gong, L.-Z. Org. Lett. 2006, 8, 6023.

[22] (a) Banerjee, T. S.; Paul, S.; Sinha, S.; Das, S. Bioorg. Med. Chem. 2014, 22, 6062 .

(b) Wishka, D. G.; Walker, D. P.; Yates, K. M.; Reitz, S. C.; Jia, S.; Myers, J. K.; Olson, K. L.; Jacobsen, E. J.; Wolfe, M. L.; Groppi, V. E.; Hanchar, A. J.; Thornburgh, B. A.; Cortes-Burgos, L. A.; Wong, E. H.; Staton, B. A.; Raub, T. J.; Higdon, N. R.; Wall, T. M.; Hurst, R. S.; Walters, R. R.; Hoffmann, W. E.; Hajos, M.; Franklin, S.; Carey, G.; Gold, L. H.; Cook, K. K.; Sands, S. B.; Zhao, S. X.; Soglia, J. R.; Kalgutkar, A. S.; Arneric, S. P.; Rogers, B. N. J. Med. Chem. 2006, 49, 4425.

[23] Liu, H.; Cun, L. F.; Mi, A. Q.; Jiang, Y. Z.; Gong, L. Z. Org. Lett. 2006, 8,6023 .

[24] Yang, H.; Carter, R. G. J. Org. Chem. 2009, 74, 5151.

[25] (a) Nath, R.; Pathania, S.; Grover, G.; Akhtar, M. J. J. Mol. Struct. 2020, $1222,128900$.

(b) Arun, Y.; Saranraj, K.; Balachandran, C.; Perumal, P. T. Eur. J. Med. Chem. 2014, 74, 50.

[26] (a) Zhang, L.-L.; Zhang, J.-W.; Xiang, S.-H.; Guo, Z.; Tan, B. Chin. J. Chem. 2018, 36, 1182

(b) Mei, G.-J.; Shi, F. Chem. Commun. 2018, 54, 6607.

(c) Tan, F.; Xiao, W.-J; Zeng, G.-P. Chin. J. Org. Chem. 2017, 37, 824 (in Chinese).

(谭芬，肖文精，曾国平，有机化学，2017, 37, 824.)

(d) Xiao, Y.-L; Zhou, Y.; Wang, J.; Wang, J.-X; Liu, H. Chin. J. Org. Chem. 2015, 35, 2035 (in Chinese).

(肖永龙, 周宇, 王江, 王进欣, 柳红, 有机化学, 2015, 35, 2035.)

[27] (a) Shi, F.; Zhu, R.-Y.; Liang, X.; Tu, S.-J. Adv. Synth. Catal. 2013, $355,2447$.

(b) Chen, X.-H.; Wei, Q.; Luo, S.-W.; Xiao, H.; Gong, L.-Z. J. Am. Chem. Soc. 2009, 131, 13819.

[28] Shi, F.; Tao, Z.-L.; Luo, S.-W.; Tu, S.-J.; Gong, L.-Z. Chem. Eur. J. 2012, 18, 6885.

[29] Tian, L.; Hu, X.-Q.; Li, Y.-H.; Xu, P.-F. Chem. Commun. 2013, 49 , 7213.

[30] Xiao, J.-A.; Liu, Q.; Ren, J.-W.; Liu, J.; Carter, R. G.; Chen, X.-Q.; Yang, H. Eur. J. Org. Chem. 2014, 2014, 5700.

[31] Ren, J.-W.; Zhou, Z.-F.; Xiao, J.-A.; Chen, X.-Q.; Yang, H. Eur. J. Org. Chem. 2016, $2016,1264$.

[32] (a) Wang, T.; Yu, Z.; Hoon, D. L.; Phee, C. Y.; Lan, Y.; Lu, Y. J. Am. Chem. Soc. 2016, 138, 265

(b) Wei, X.; Liu, D.; An, Q.; Zhang, W. Org. Lett. 2015, 17, 5768.

(c) Wang, B.; Tu, Y. Q. Acc. Chem. Res. 2011, 44, 1207

[33] (a) Alba, A. N.; Rios, R. Chem. Asian. J. 2011, 6, 720.

(b) Fisk, J. S.; Mosey, R. A.; Tepe, J. J. Chem. Soc. Rev. 2007, 36, 1432 .

[34] Zhang, J.; Liu, X.; Wu, C.; Zhang, P.; Chen, J.; Wang, R. Eur. J. Org. Chem. 2014, 2014, 7104.

[35] Wei, Y.; Liu, Z.; Wu, X.; Fei, J.; Gu, X.; Yuan, X.; Ye, J. Chem. Eur. J. 2015, 21, 18921 .

[36] Zhang, S.-Y.; Ruan, G.-Y.; Geng, Z.-C.; Li, N.-K.; Lv, M.; Wang, 
Y.; Wang, X.-W. Org. Biomol. Chem. 2015, 13, 5698.

[37] Wang, C.-M.; Xiao, J.-A.; Wang, J.; Wang, S.-S.; Deng, Z.-X.; Yang, H. J. Org. Chem. 2016, 81, 8001.

[38] (a) Gabriel, I. Molecules 2020, 25, 1480.

(b) Gensicka-Kowalewska, M.; Cholewiński, G.; Dzierzbicka, K. RSC Adv. 2017, 7, 15776.

[39] (a) Mayekar, N. V.; Nayak, S. K.; Chattopadhyay, S. Synth. Commun. 2004, 34, 3111.

(b) Jacob, R. G.; Perin, G.; Botteselle, G. V.; Lenardão, E. J. Tetrahedron Lett. 2003, 44, 6809.

[40] (a) Chen, M.-W.; Cai, X.-F.; Chen, Z.-P.; Shi, L.; Zhou, Y.-G. Chem. Commun. 2014, 50, 12526. (b) Dickmeiss, G.; Jensen, K. L.; Worgull, D.; Franke, P. T.; Jorgensen, K. A. Angew. Chem. Int. Ed. 2011, 123, 1681.

[41] Li, S.; Wang, J.; Xia, P.-J.; Zhao, Q.-L.; Wang, C.-M.; Xiao, J.-A.; Chen, X.-Q.; Xiang, H.-Y.; Yang, H. J. Org. Chem. 2018, 83, 12284.

[42] Ren, J.-W.; Wang, J.; Xiao, J.-A.; Li, J.; Xiang, H.-Y.; Chen, X.-Q.; Yang, H. J. Org. Chem. 2017, 82, 6441.

[43] Ren, J.-W.; Zheng, L.; Ye, Z.-P.; Deng, Z.-X.; Xie, Z.-Z.; Xiao, J.-A.; Zhu, F.-W.; Xiang, H.-Y.; Chen, X.-Q.; Yang, H. Org. Lett. 2019, 21, 2166.

[44] Wang, J.; Deng, Z.-X.; Wang, C.-M.; Xia, P.-J.; Xiao, J.-A.; Xiang, H.-Y.; Chen, X.-Q.; Yang, H. Org. Lett. 2018, $20,7535$.

(Fan, Y.) 